Check for updates

Cite this: Mater. Adv., 2021, 2, 7731

Received 20th August 2021 Accepted 29th September 2021

DOI: 10.1039/d1ma00747e

rsc.li/materials-advances

\section{Nanoarchitectonics of metal organic frameworks and PEDOT layer-by-layer electrodes for boosting oxygen reduction reaction $\dagger$}

\author{
Gonzalo E. Fenoy, (D ab Matías Rafti, (D) ${ }^{a}$ Waldemar A. Marmisollé (iD *a and \\ Omar Azzaroni id a
}

\begin{abstract}
We present a strategy for the integration of three building blocks in a functional interfacial nanoarchitecture in order to fabricate composite films with improved features towards the electrochemical oxygen reduction reaction (ORR). Firstly, multilayer films of polyethyleneimine (PEI) and poly(3,4ethylenedioxythiophene):poly(4-styrenesulfonate) (PEDOT:PSS) complex are generated using the layerby-layer method by making use of the electrostatic interactions between the cationic polyelectrolyte and the negatively charged colloidal polymeric complex. Film assembly shows an exponential growth up to 10 bilayers, with a sharp increase in the amount of deposited material above 4 bilayers. While the electrical connection through the LbL assembled films up to 10 bilayers is efficient, optimal electrochemical performance of the modified electrodes towards the ORR in neutral solutions occurs for 4 bilayers, and then decreases gradually for 6 and 10 bilayers. Subsequently, in order to explore the effect of MOF inclusion on this architecture, Zn-based ZIF-8 nanocrystallites were deposited on the electrodes previously modified with LbL assemblies of 4-bilayers. The micro/mesoporosity, thus added to the films, resulted in an improvement in the ORR performance which can be understood by considering the already demonstrated ability of ZIF-8 for selective oxygen adsorption from solution. Furthermore, ZIF- 8 inclusion does not interfere with the ionic transport through the composite film, which is crucial for efficient electrocatalysis. The hereby presented study exemplifies a simple approach for the fabrication and optimization of complex functional interfaces with improved activity towards ORR in neutral $\mathrm{pH}$ aqueous environments.
\end{abstract}

\section{Introduction}

The rapidly rising energy demand together with an ever increasing global population threatens the environment due to economic expansion powered by fossil fuels; this causes among other concerning effects, climate change, energy shortage, and environmental disruption. ${ }^{1,2}$ The current increase in the concentration of atmospheric carbon dioxide $\left(\mathrm{CO}_{2}\right)$ is believed to exhibit a deep correlation with the global temperature rise, which demands the emergence of novel sustainable and environmentally friendly technologies for energy conversion and storage. ${ }^{3}$ In this way, proton exchange membrane fuel cells (PEMFCs) have received much attention both from the

\footnotetext{
${ }^{a}$ Instituto de Investigaciones Fisicoquímicas Teóricas y Aplicadas (INIFTA), Departamento de Química, Facultad de Ciencias Exactas, Universidad Nacional de La Plata (UNLP), CONICET. 64 and 113, La Plata (1900), Argentina. E-mail: wmarmi@inifta.unlp.edu.ar

${ }^{b}$ Instituto de Investigación e Ingeniería Ambiental, Universidad Nacional de San Martín, 25 de mayo y Francia, 1 piso, San Martin 1650, Buenos Aires, Argentina $\dagger$ Electronic supplementary information (ESI) available. See DOI: 10.1039/d1ma00747e
}

academic and industrial perspectives as they exhibit considerable energy conversion efficiency, high energy density, low to zero pollution emission, and high reliability, making them promising candidates for use in devices for sustainable and clean energy production. ${ }^{4}$ Additionally, the incipient metal-air batteries, such as $\mathrm{Zn}$-air batteries, are cost-effective and exhibit high energy density, and thus are also considered for applications related to energy storage with renewable power sources. ${ }^{5}$ In both metal-air batteries and PEMFCs, the cathodic oxygen reduction reaction (ORR) constitutes the core of the electrochemical conversion processes, and consequently, the performance of the considered device will depend mainly on the features of the ORR electrocatalysts employed. ${ }^{6}$ Therefore, the development of efficient and cost-effective ORR electrocatalysts with enhanced activity and durability is crucial for the extensive employment of these energy conversion and storage technologies. $^{7-9}$

On the other hand, nanoarchitectures presenting the integration of different materials into structurally stable and functional composites have received attention from the scientific 
community, as they have showed great versatility in applications related to energy storage/conversion, catalysis, and biosensor technologies. ${ }^{10-12}$ Research efforts employing such approach are often referred to as "nanoarchitectonics", a term popularized by Ariga and co-workers. ${ }^{13}$ Within nanoarchitectonics, the accurate manipulation of film architecture and the successful incorporation of different building blocks are key aspects for the fabrication of electrochemical devices. In this sense, layer-by-layer (LbL) assembly becomes a straightforward methodology which allows the integration of polymers and other nanomaterials in a controlled fashion, avoiding undesirable effects such as aggregation and enabling intimate contact between film constituting elements, crucial for transport properties. ${ }^{14-16}$ Usually, multilayer composite coatings are obtained by sequential deposition of materials with particular features that allow specific interactions and enable the emergence of functional properties. ${ }^{17-19}$ In this regard, the construction of multilayer thin-film architectures was already shown to be possible by resorting to hydrogen bonding, electrostatic interactions, and/or van der Waals "soft" interactions. $^{20-22}$ Aside from the above-mentioned energyrelated applications, the use of functional multilayer coatings in diverse fields, such as drug delivery, biosensing, catalysis, lithography, and electronic signaling and memory, ${ }^{16,23-27}$ has also been reported.

Particularly, multilayer coatings have arisen as promising candidates for use in fuel cell technology due to the possibility of straightforward architecture control with nanometric precision on the thickness and the integration of diverse elements and their functional properties. Conceptually, a multilayer assembly is appealing when considering fuel cell catalyst layers, since the catalyst material will be intimately packed with the host matrix, yielding pre-designed fuel permeability and reduced catalyst loss. ${ }^{24,28,29}$ In this respect, electrolyte or mass transfer $\left(\mathrm{O}_{2}\right.$ diffusion $)$ resistance is a crucial factor which has a huge impact on the performance of PEMFCs and metal-air batteries, and, consequently, should not be neglected..$^{8,30}$

On the other hand, conducting polymers (CPs) are a class of materials which have been extensively employed in applications related to recyclable power sources and energy storage devices. ${ }^{31}$ Among them, poly(3,4-ethylenedioxythiophene) (PEDOT) is among the most studied (and actually employed) materials because of its stability, outstanding electronic and optical features, and well-known production methods. ${ }^{32}$ Moreover, it was recently shown that PEDOT-based materials present excellent values for mixed ionic-electronic conductivity, thus having a great potential for applications related to fuel cells and metal-air batteries. ${ }^{33-35}$ The use of PEDOT as electrocatalyst for the ORR has been a subject of intense research since Winther-Jensen et al.'s pioneering work. ${ }^{36}$ Despite the fact that, in some reported composite electrodes, PEDOT could be considered merely as a conducting binder while the metal impurities usually present can account for the observed electrocatalytic effect, the electrocatalytic activity of metal-free PEDOT has been unambiguously proved in recent studies. ${ }^{37,38}$ Moreover, it has been recently reported that the ORR reaction catalyzed by PEDOT occurs preferentially through the 4-electron pathway with water as the product. ${ }^{34}$ The integration of PEDOT in multilayer assemblies (mainly in the form of poly $(3,4-$ ethylenedioxythiophene):poly(4-styrenesulfonate), PEDOT:PSS) has been employed as an effective approach towards the combining of features from conducting polymers and other materials, such as polyelectrolytes, noble metal nanoparticles and UV-absorbent materials. ${ }^{24,26,39,40}$ Particularly, the integration of electrocatalytic components in PEDOT films has been an extensively employed strategy for increasing the efficiency towards the ORR. In this regard, a wide variety of materials have been reported to decrease the ORR onset potential or enhance the 4-electron mechanism when integrated to PEDOT-based electrodes, including $\mathrm{CoMn}_{2} \mathrm{O}_{4}$ spinel nanorods, ${ }^{41}$ reduced graphene oxide with $\mathrm{Fe}_{3} \mathrm{O}_{4}$ nanorods ${ }^{42}$ and $\mathrm{Au}$ nanoparticles, ${ }^{43}$ graphene quantum dots, ${ }^{44}$ hemin, ${ }^{45}$ $\mathrm{MnO}_{x}{ }^{46}$ and $\mathrm{PdO}_{x}{ }^{47}$ In addition, the integration of electrochemically inactive materials such as PEG-PDMS-PEG block copolymers within the PEDOT matrix has been reported to improve the ORR by modifying the conducting polymer structure and, thus, its electrocatalytic activity. ${ }^{48}$ These types of strategies operate on the redox reaction mechanism of the ORR.

Metal-organic frameworks (also known as MOFs) and MOFderived materials have recently attracted a great deal of attention due to interesting features such as chemical versatility, tunable porosity and catalytic and electrocatalytic activity. ${ }^{49-52}$ Moreover, the integration of MOFs in hybrid architectures has been shown as a feasible and effective strategy to improve the ORR performance of composite electrode materials. To date, several MOF-based composites with polymers, nanocarbons and even nanoparticles have been reported. ${ }^{53-55}$ Particularly, when dealing with the combination of MOFs and polymers, many studies have focused on possible synthesis pathways based on existing interactions. Diverse successful strategies for this end have been reported recently, involving the synthesis of mixed matrix membranes (MMMs), polymers grafted-from or grafted to MOF particles, polymer-templated MOFs, and MOF grown from polymer films used as primers. ${ }^{54,56,57}$ In this regard, the integration of MOF (ZIF-67) in PEDOT has been previously reported as a precursor material for preparing a Co-N-S tripledoped porous carbon ORR catalyst by calcination. ${ }^{58}$

Along the same line of thought, and encouraged by the many proposed examples of composites exploiting the advantages of including MOFs as selective gas adsorbers which would enable substantial performance improvements (e.g., carbon dioxide reduction reaction ${ }^{59-61}$ or reduction reactions enhanced by increased $\mathrm{H}_{2}$ storage ${ }^{62-64}$ ), we have recently demonstrated a different mechanism for improving the ORR on conducting polymer electrodes based on the $\mathrm{O}_{2}$ preconcentration within the MOF structure in neutral solutions. In this strategy, the inclusion of ZIF-8 MOF (an archetypal member of the zeolitic imidazolate subclass, composed of tetrahedrally coordinated $\mathrm{Zn}^{2+}$ ions with 2-methylimidazole bidentate linkers) ${ }^{65}$ on top of a conducting polymer-modified electrode enhances the ORR performance by increasing $\mathrm{O}_{2}$ availability. ${ }^{57,66}$ As ZIF-8 is an insulating electrochemically inactive material, the enhancement is not related with the ORR redox mechanism but with the $\mathrm{O}_{2}$ transport. Furthermore, polymer capping adds a further degree of customizability to these ZIF-8 MOF colloidal units; 
e.g., poly(allylamine hydrochloride)-capped ZIF-8 nanocrystals have been successfully incorporated into polyelectrolyte-conducting polymers complex films via LbL, improving also performance towards ORR. ${ }^{67}$

Herein, we show the successful integration of ZIF-8 on LbL assemblies prepared using PEI and PEDOT:PSS in order to optimize electrodes for ORR in neutral solutions. We make use of the sulfonate moieties exposed from PEDOT:PSS in order to incorporate ZIF-8 and we show that, while the deposition of more than 4 bilayers deteriorates the performance of the multilayer-modified electrodes, the incorporation of ZIF-8 brings the important feature of porosity and allows $\mathrm{O}_{2}$ preconcentration within the films, optimizing the electrocatalytic activity of the nanostructured electrode material.

\section{Materials and methods}

\subsection{Reagents}

Polyethylenimine $\left(M_{\mathrm{W}} 10 \mathrm{kDa}\right)$ (PEI), PEDOT:PSS $(1.3 \% \mathrm{w} / \mathrm{w})$, 4-(2-hydroxyethyl)-1-piperazineethanesulfonic acid (HEPES), $\mathrm{Zn}\left(\mathrm{NO}_{3}\right)_{2} \cdot 6 \mathrm{H}_{2} \mathrm{O}$, graphite rods (diameter $=3 \mathrm{~mm}$, length = $150 \mathrm{~mm}$ ) and 2-methyl-imidazole (2m-Im) were purchased from Sigma-Aldrich. KCl was obtained from Anedra. Methanol was obtained from Dorwil. All chemicals were used as received without further purification. All aqueous solutions were prepared using Milli-Q water.

\subsection{Instrumentation}

Calculated X-ray diffraction patterns were obtained from Cambridge Crystallographic Data Base (CCDB) using already reported ZIF-8 structures with Mercury ${ }^{\circledR}$ software. Experimental $\mathrm{XRD}$ patterns corresponding to the synthesized films were collected at room temperature under ambient conditions using a Phillips X'Pert diffractometer.

UV-Vis spectra were acquired using a UV-Vis Agilent $8453 \mathrm{E}$ spectrometer employing quartz substrates.

FTIR spectra were acquired in the ATR mode (MIRacle ${ }^{\mathrm{TM}}$ Single Reflection accessory equipped with a diamond prism) using a FTIR Nicolet 480 spectrometer. 128 scans were taken between 500 and $4000 \mathrm{~cm}^{-1}$ with a resolution of $4 \mathrm{~cm}^{-1}$.

Electrochemical measurements were carried out using a Gamry Reference 600 potentiostat. The experimental setup consisted of a three-electrode PTFE-lined cell with $2 \mathrm{~mL}$ volume capacity. The counter electrode was a Pt wire (or a graphite rod when explicitly stated), and $\mathrm{Ag} / \mathrm{AgCl}(3 \mathrm{M} \mathrm{NaCl})$ was used as the reference electrode. All reported potentials are referred to this electrode. Gold electrodes were prepared by sputtering Au on a glass plate. A thin layer of Ti was previously deposited on the glass substrates in order to improve adhesion.

Oxygen reduction tests were performed in HEPES $5 \mathrm{mM}$ $0.1 \mathrm{M} \mathrm{KCl}$ solution ( $\mathrm{pH} 7$ ). Oxygen or nitrogen-saturated solution was obtained by bubbling pure gas for 30 minutes previous and also during the electrochemical experiments.

Quartz crystal microbalance (QCM) measurements were performed with a QCM200 setup (Stanford Research Systems) using gold-coated quartz sensors (QCM25 $5 \mathrm{MHz}$, sensitivity factor: $56.6 \mathrm{~Hz} \mathrm{~cm}{ }^{2} \mu \mathrm{g}^{-1}$ ). Experiments were performed with quiescent solutions in a cell at room temperature.

Scanning electron microscopy images of the compositecoated Au substrates were acquired using a FEI Quanta 200 apparatus under vacuum conditions (Servicio de Microscopía Electrónica de Barrido y Microanálisis del LIMF - Fac. de Ingeniería - UNLP).

\subsection{Substrate modification}

The LbL assembly was started with the deposition of a PEI layer, as this polymer is known to promote adhesion on different substrates such as $\mathrm{Au}$, glass and quartz. ${ }^{22}$ In order to achieve this goal, the substrates were immersed in a $1 \mathrm{mg} \mathrm{mL}{ }^{-1}$ PEI solution for 10 minutes and washed two times for 3 minutes with Milli-Q water. The assembly procedure was continued by immersing the substrates into a PEDOT:PSS 1/10 (Milli-Q water) solution for 10 minutes and washing two times with Milli-Q water (3 minutes each). The whole described procedure constituted the assembly of one bilayer. Then, the deposition cycle was repeated $n$ times for building the desired number of bilayers.

For ZIF-8 deposition, the substrates were placed in a cell which was filled with a fresh mixture of $\mathrm{Zn}\left(\mathrm{NO}_{3}\right)_{2} \cdot 6 \mathrm{H}_{2} \mathrm{O}$ $(25 \mathrm{mM})+2$-methyl-imidazole $(50 \mathrm{mM})$ stock methanolic solutions at room temperature. The substrates were then rinsed with fresh methanol and dried under $\mathrm{N}_{2}$ prior to the next MOF deposition cycle. This procedure was repeated until a suitable MOF film thickness was obtained (the films obtained after successive depositions will be referred as $m \times$-ZIF-8, where $m$ is the number growth cycles carried).

\section{Results and discussion}

\subsection{LbL assembly growth}

The optimization of the ORR through the integration of the MOF in the electroactive polymeric film was performed in two deposition sequences. The first step involved the building up of multilayer assemblies onto different substrates, using polyelectrolytes as the building blocks: the negatively charged PEDOT:PSS dispersions and the cationic polyelectrolyte PEI. Later, the incorporation of the PEDOT:PSS complex not only brings the electrocatalytic functionality of PEDOT but also provides the conducting paths throughout the interfacial architecture, while PEI acts as the necessary counterpart in the assembly. A schematic representation of the LbL assembly process is shown in Fig. 1A.

In order to study the LbL construction process, we followed the growth of the assemblies on quartz substrates by UV-VIS spectroscopy. Particularly, the absorption ascribed to the styrene sulfonate moieties at $226 \mathrm{~nm}$ was monitored as the number of bilayers increased. ${ }^{39}$ From Fig. $1 \mathrm{~A}$ and $\mathrm{B}$ it can be seen that the assembly process shows an exponential region, with a slower (linear) building up until 4 bilayers. The occurrence of an exponential building-up of LbL assemblies of PEDOT:PSS with PEI has 
A
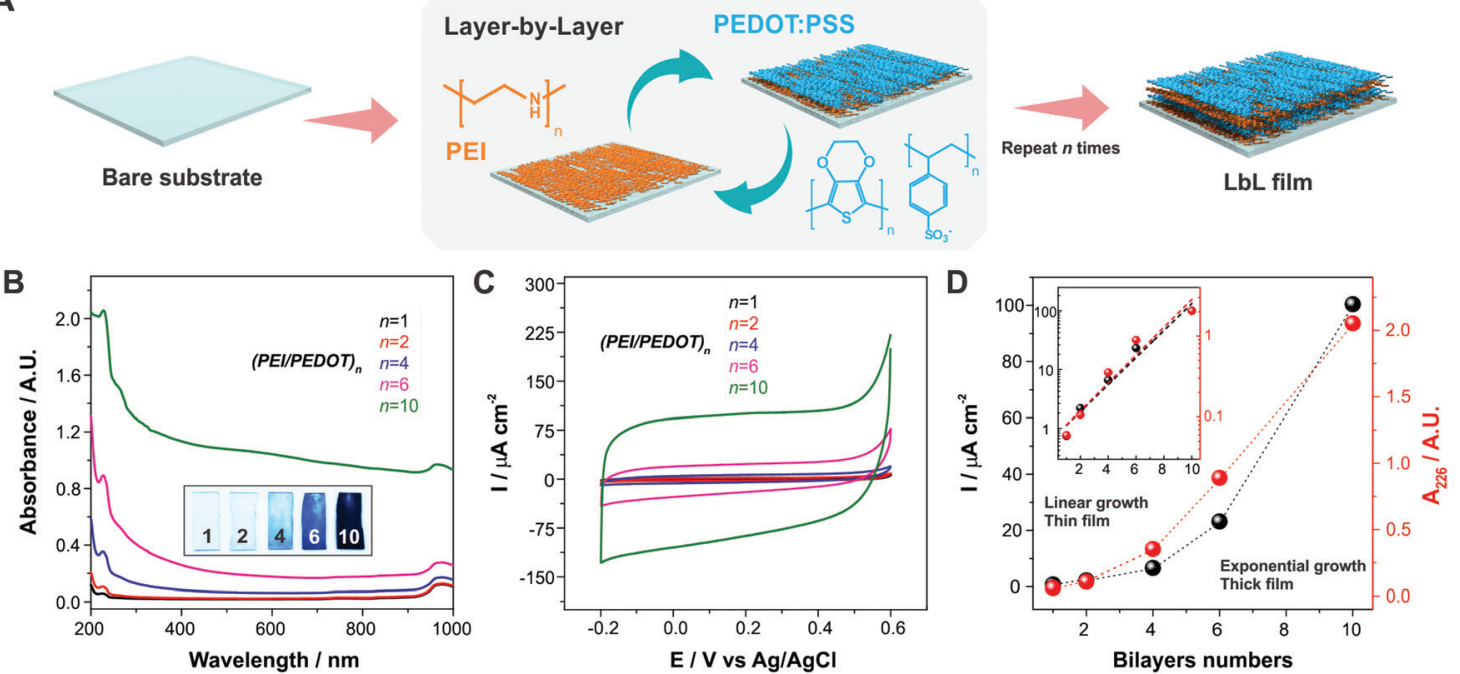

Fig. 1 (A) Schematic illustration of the LbL assembly process. (B) UV-Vis spectra and pictures of the assemblies with different numbers of bilayers deposited on quartz substrates. (C) Cyclic voltammetry curves of assemblies with different numbers of bilayers, scan rate $200 \mathrm{mV} \mathrm{s}{ }^{-1}, 0.1 \mathrm{M} \mathrm{KCl}$. (D) Current at $0.2 \mathrm{~V}$ and absorbance at $226 \mathrm{~nm}$ of assemblies with different numbers of bilayers together with the exponential fitting of both data. The inset shows the data in a logarithm scale and the linear fitting of both measurements.

been already reported. ${ }^{26,68}$ Moreover, from the images presented in Fig. 1B it can be noticed that the color of films assembled becomes increasingly intense as the number of bilayers increases, which implies that PEDOT is continuously incorporated in the films as the amount of material increases. This is not a trivial result, as it was previously reported that PEDOT (a positively charged conducting polymer) can be lost along the film assembly process due to the suppression of electrostatic forces between the cationic polyelectrolyte and PSS, which is also surrounding the conducting polymer PEDOT, which consequently would compromise the stability of the assemblies. ${ }^{69}$ Furthermore, this would also have implications on the retention of electrochemical connectivity throughout the successive layers of the film (see Fig. 1C): i.e., the incorporation of two polyelectrolytes without any conducting material in thin films electrodes would result in the blockage of the electrode surface. ${ }^{15}$ On the other hand, the preservation of an electroactive polymeric framework within the films as the assembly process progresses results in an increased electrocatalytic activity (until the occurrence of a blockage when a certain limiting thickness is surpassed, see the results in ORR section below).

In order to study the electrochemical connection of the assembled film on gold-coated glass substrates during the consecutive LbL procedures, we performed cyclic voltammetry (CV) measurements in a potential region where no electrocatalytic reaction is known to occur $\left(-0.2 \mathrm{~V}\right.$ to $\left.0.6 \mathrm{~V}, 200 \mathrm{mV} \mathrm{s}^{-1}\right)$, see Fig. 1D. The CV curves have a rectangular shape in the potential range, ascribed to the capacitance during charging/discharging due to the high amount of polyelectrolyte incorporated in the assemblies, as it was reported for similar systems. ${ }^{70,71}$

It is well-known for LbL assemblies that the successive deposition of layers can lead to a blocking of ionic and/or charge transport across the assembly, particularly when both the components are not electronically well-connected. ${ }^{15,16}$ In this sense, it can be observed in Fig. $1 \mathrm{C}$ and $\mathrm{D}$, that the current density at $0.2 \mathrm{~V}$ increases with the number of bilayers deposited following the same exponential growth as the UV-Vis absorption at $226 \mathrm{~nm}$ (see the inset in which similar linear fits can be obtained when logarithmic scales are used for both absorption and current plots). As the integrated voltammetric charge (or equally in this case, the capacitive current) is indicative of the amount of electrochemically active material deposited after each functionalization cycle, it can be concluded that all the deposited material is linked throughout the interfacial nanoarchitecture at least up to 10-bilayer assembly, showing an efficient electrochemical connection without blockage of the ionic/electronic transport across the film even at a high scan rate of $200 \mathrm{mV} \mathrm{s}^{-1}$. In this case, the upper layers are still connected with the lower ones, contrary to what is observed when dealing with thick-film electrodes..$^{15}$ It can be concluded then, that incorporation of PEDOT:PSS brings the necessary electronic conductivity to the assemblies, whereas its colloidal nature allows for a straightforward integration strategy and an accurate thickness control of the assemblies. Finally, the presence of exposed sulfonate moieties on the film also allows for the efficient anchoring of an MOF film on top of this polymeric coating (see next section).

\subsection{ZIF-8 deposition on the LbL assemblies}

We propose the deposition of ZIF-8 MOF units onto LbL-modified substrates as a strategy for increasing the overall performance towards ORR. Our hypothesis is based on the already demonstrated capability of ZIF-8 to act as a porous reservoir for molecular oxygen. In this sense, we performed the deposition of ZIF-8 via sequential growth cycles from methanolic precursor solutions onto the LbL assemblies of 4-bilayer-modified Au substrates 
A

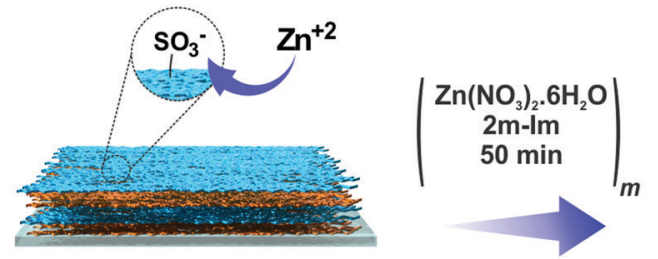

LbL thin film

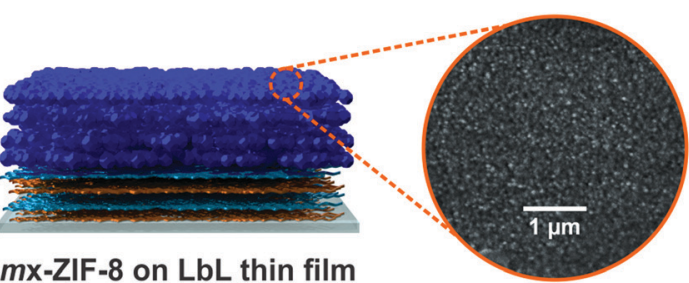

B

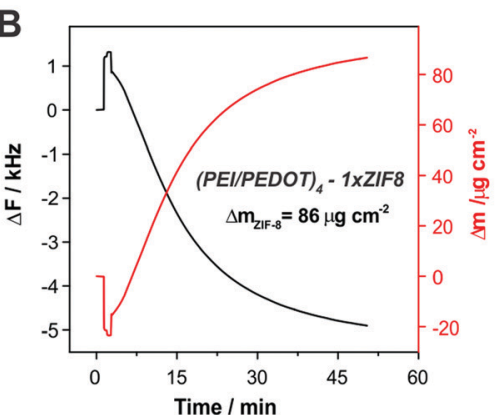

C

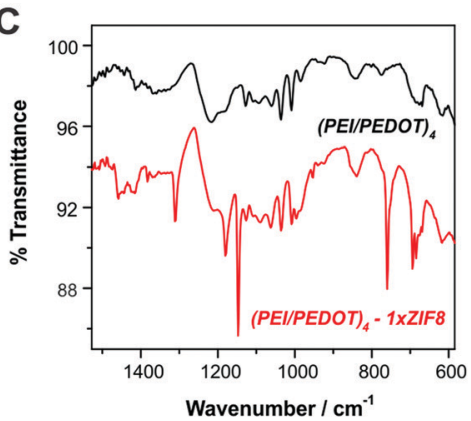

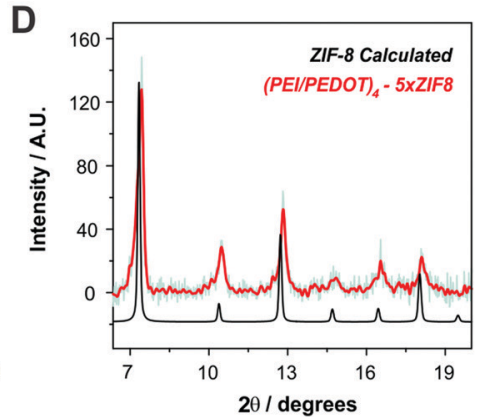

Fig. 2 (A) Schematic showing the deposition of the MOF films onto the LbL-modified substrates, together with a SEM picture of a $3 \times Z$ ZIF-8-(PEI/ PEDOT) 4 substrate. (B) Frequency and mass changes measured by QCM for one ZIF- 8 deposition cycle onto a 4 bilayer-modified gold substrate. (C) ATRFTIR spectra of a (PEI/PEDOT) ${ }_{4}$-modified gold substrate before and after one ZIF-8 deposition cycle. (D) XRD pattern (raw: light green; smoothed: red) of a $5 \times$ ZIF-8-(PEI/PEDOT) ${ }_{4}$-modified substrate.

((PEI/PEDOT $\left.)_{4}\right)$, as shown in Fig. 2A. In this way, and in order to provide a suitable primer for the MOF layer growth, LbL assemblies were always terminated with a layer of PEDOT:PSS, which exposes sulfonate moieties which were used previously as ZIF-8 surface-confined nucleation promoter.

Each ZIF-8 growth cycle was performed for 50 minutes ( $m \times$-ZIF-8, where $m$ is the number of cycles), and the deposition was monitored in situ by QCM, following a previously reported procedure. ${ }^{57}$ In Fig. $2 \mathrm{~B}$ the change in surface mass measured by QCM for a $1 \times$-ZIF-8 growth cycle onto a 4 bilayer LbL-substrate is shown. The deposited mass obtained by QCM for a $1 \times$-ZIF-8 film growth cycle is higher than values reported in recent works (see Table S1, ESI $\dagger$ for comparison with the recently reported methods)..$^{56,57,66,72}$ This result can be explained by taking into account the high affinity of $\mathrm{Zn}^{2+}$ ions for the exposed sulfonate moieties present in the PEDOT:PSS layer. It has been demonstrated that the interactions between MOF precursors and surface-exposed moieties are crucial to manipulate the dynamics of film growth. In this sense, the growth of ZIF-8 films on Au substrates functionalized with selfassembled monolayers (SAMs) bearing different moieties was studied by Tuninetti et al., showing that sulfonate moieties promote an enhanced deposition of ZIF-8 films with low intergrain mesoporosity when compared with other primers. ${ }^{56}$ Moreover, Bindini et al. showed that the use of a sulfonate-bearing polyelectrolyte brush as an anchoring platform (a 3d-primer rather than a surface confined 2D-primer) increased the amount of ZIF-8 deposited compared to what could be expected from a sulfonate-terminated SAM. ${ }^{72}$ In that work, it was stated that the brushes contain a higher density of sulfonate moieties that are able to coordinate $\mathrm{Zn}^{+2}$ ions available from the solution, which subsequently will play a decisive role as nucleation sites for the ZIF-8 film. In a similar fashion, the high concentration of sulfonate moieties available in the PEDOT:PSS films employed here induces the deposition of high amounts of ZIF-8 on the LbL assemblies (using the same deposition conditions as those observed for sulfonate-bearing polymer brushes) but in a shorter period of time. This result implies that the use of a simple strategy can produce efficient anchoring of an MOF layer without the need of complex synthetic procedures, as those required, for example, for surface-initiated atom transfer radical polymerization (SI-ATRP) employed in the abovementioned approach.

In order to confirm the effective deposition of the MOF film onto the LbL-modified substrates, we performed ATR-FTIR measurements. Fig. 2C shows the ATR-mode FTIR spectra of a (PEI/PEDOT) $)_{4}$-modified substrate before and after the deposition of a $1 \times$-ZIF- 8 film. The FTIR spectrum of the LbL-coated Au substrate shows the typical bands of PEDOT:PSS, which are in line with those reported before. ${ }^{73}$ Briefly, the spectrum shows vibrational signals related to sulfonate moieties from PSS: a doublet positioned at 1120 and $1180 \mathrm{~cm}^{-1}$ corresponding to the asymmetric stretching mode and a band at $1037 \mathrm{~cm}^{-1}$ ascribable to the symmetric stretching mode. The band at about $1009 \mathrm{~cm}^{-1}$ has been assigned to in-place aromatic $\mathrm{CH}$ bending. Finally, the band at $840 \mathrm{~cm}^{-1}$ corresponds to the outof-plane aromatic $\mathrm{CH}$ deformation. ${ }^{22}$ After 1 cycle of MOF deposition, the spectrum also shows the typical features of ZIF-8, most of them corresponding to imidazole ring vibrations, namely, the intense band in the region between $1350 \mathrm{~cm}^{-1}$ and $1500 \mathrm{~cm}^{-1}$ corresponding to the entire imidazole ring stretching mode, the bands in the $900-1350 \mathrm{~cm}^{-1}$ region arising from in-plane ring bending, and bands appearing below $800 \mathrm{~cm}^{-1}$ corresponding to out-of-plane bending. ${ }^{57,66}$ The above described 
results confirm the effective anchoring of the MOF layer onto the LbL-modified substrates.

Furthermore, a SEM image of a $3 \times$-ZIF-8-(PEI/PEDOT) $)_{4}$ substrate is shown in Fig. 2A, where a top view of a film showing the nanocrystals of the deposited MOF can be seen, as previously reported. ${ }^{74,75}$ When compared with the SEM picture of the (PEI/PEDOT) $)_{4}$ substrate (Fig. S1, ESI $\dagger$ ), the differences ascribed to the deposited MOF layer are clearly evidenced.

In order to corroborate that the structure of the layer grown features the crystalline structure corresponding to ZIF-8, XRD measurements were performed. Fig. 2D shows the XRD pattern of a $5 \times$-ZIF.8-(PEI/PEDOT) $)_{4}$-modified electrode together with the calculated pattern for ZIF-8 from the reported structure. In this case, a $5 \times$-ZIF- 8 modification was needed onto the LbLmodified substrate in order to acquire the necessary thickness for the diffraction peaks to be detected in the experimental configuration employed. The peaks belonging to (110), (200), (211) and (222) Miller indices, appearing at $7.3^{\circ}, 10.4^{\circ}, 12.7^{\circ}$ and $18.0^{\circ}$ respectively, can be observed in the MOF-modified substrate. This result confirms the effective anchoring and, more importantly, the crystalline structure of the deposited porous ZIF-8 film onto the multilayer thin films. ${ }^{57,66}$

\subsection{ORR electroactivity}

We explored the electroactivity of the LbL assemblies of PEI/PEDOT:PSS towards the ORR in neutral solutions for an increasing number of LbL-deposition cycles. The voltammetric responses towards ORR in air-bubbled $\mathrm{pH} 7$ buffer are shown in Fig. 3A. In order to quantify the effect of the electroactive composite film thickness, the integrated charge corresponding to the ORR voltammetric wave ( $Q_{\text {ORR }}$ ) was computed (Fig. 3B). It can be observed that the $Q_{\mathrm{ORR}}$ increases with the number of bilayers up to 4 , where the value reaches a maximum; moreover, with two additional bilayers the $Q_{\text {ORR }}$ shows a small decrease. The effect becomes even more noticeable when 10 bilayers are assembled. In this sense, even when $Q_{\text {ORR }}$ shows a small increase for 10-bilayers compared to 4 and 6 bilayers (Fig. S2, ESI $\dagger$ ), the capacitive contribution to both computed parameters must be considered, ascribed to the higher amount of material deposited on the electrode surface. Thus, when performing a baseline correction, we can notice that the ORR charge for the 10 bilayer assembly diminishes compared to that observed for 4 and 6 bilayer assemblies. This result implies that, in the assemblies up to 4 bilayers, the oxygen reduction occurs throughout almost all the film under the present experimental conditions. However, when more than 4 bilayers are assembled, it is likely that the outermost layers would block oxygen diffusion. The previously commented CV experiments prove that there is electrical connectivity throughout the film, and that the potential sweep rate used is slow enough to completely reduce the film. Then, for the ORR applied potential range, the reaction is limited by diffusive transport of the required reagents probably due to slow $\mathrm{O}_{2}$ diffusion across
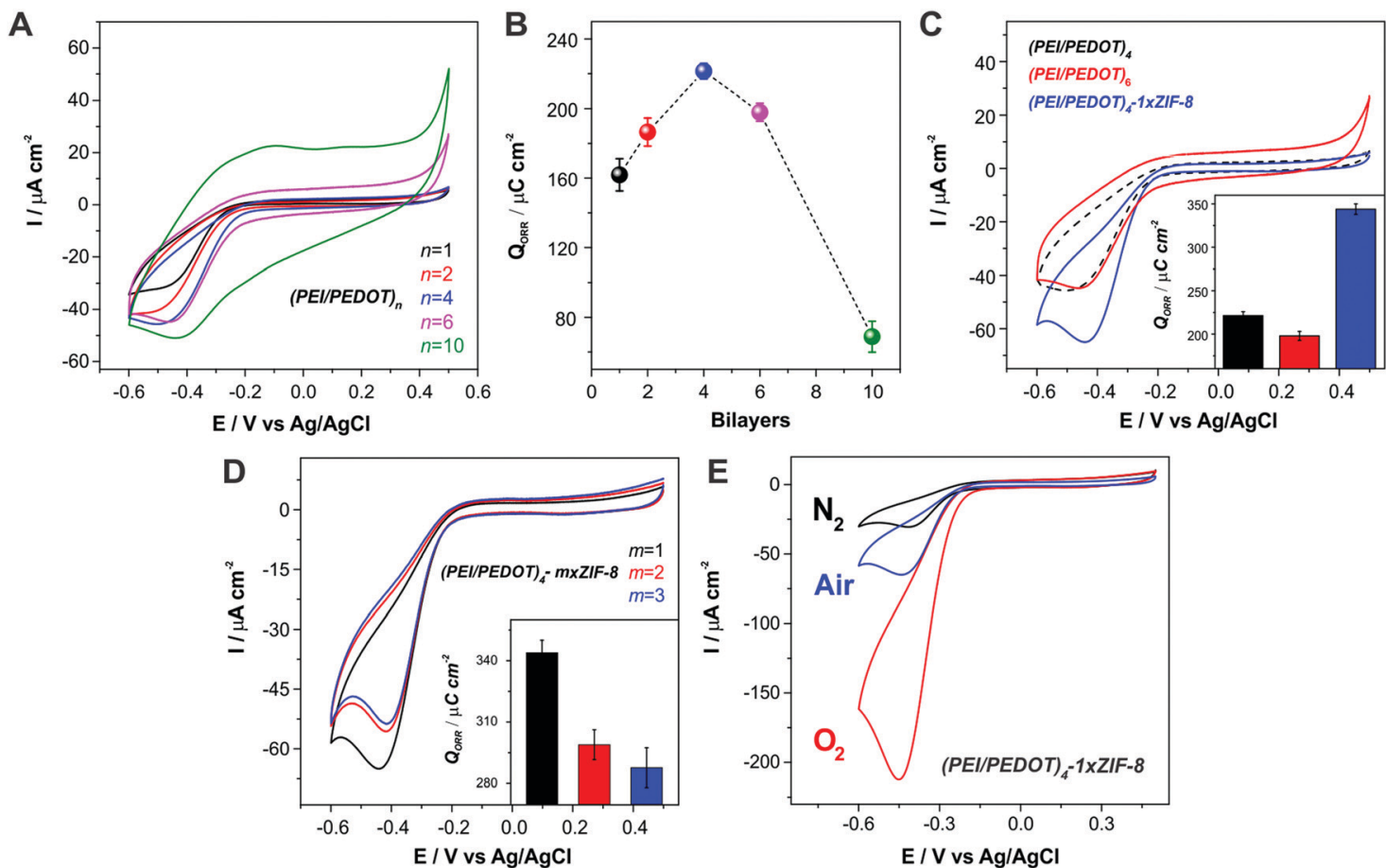

Fig. 3 (A) ORR CV curves for LbL assemblies with increasing number of bilayers (A) and the obtained ORR charge $(n=3)$. (B) ORR CV curves comparing a 4-bilayer assembly after the assembly of 2 more bilayers or the deposition of a $1 \times Z$ IF- 8 layer (the inset shows the integrated charge, $n=3$ ). (C) ORR cyclic voltammograms for increasing number of MOF layers $(m)$ onto a 4-bilayer assembly (the inset shows the integrated charge, $n=3$ ). (D) All the measurements were performed in air-saturated $5 \mathrm{mM}$ HEPES $0.1 \mathrm{M} \mathrm{KCl}$ solution; $v=10 \mathrm{mV} \mathrm{s}{ }^{-1}$. (E) ORR cyclic voltammograms for a (PEI/PEDOT) ${ }_{4}^{-}$ $1 \times \mathrm{ZIF}-8$ electrode in $\mathrm{N}_{2}-$, air- and $\mathrm{O}_{2}$-saturated $5 \mathrm{mM}$ HEPES $0.1 \mathrm{M} \mathrm{KCl}$ solutions; $v=10 \mathrm{mV} \mathrm{s}^{-1}$. 
the film. In other words, we observe that the diffusion of reactive species from the bulk solution is not equally efficient as the number of bilayers is increased. This result is also consistent with the observed abrupt increase in film thickness after 4 deposition cycles, as discussed in Section 3.1. In this regard, similar results for the oxygen reduction reaction were observed in other LbL assemblies, where oxygen diffusion was observed to become a limiting factor for more than 3 assembled bilayers. $^{76}$

Within this context, we explored the effect of incorporation of the $\mathrm{O}_{2}$-adsorbing ZIF-8 MOF on top of the electroactive film on the ORR performance of the assembled films. Fig. 3C shows that, after the deposition of a ZIF-8 layer onto the (PEI/ PEDOT) $)_{4}$-modified substrate, the voltammetric $\mathrm{O}_{2}$ reduction wave experiences a notorious increase. In this case, although the porous MOF layer is not electroactive, it acts as a high surface area oxygen reservoir. This result is in line with those previously reported for ZIF-8 films deposited on other conducting polymers: instead of decreasing the ORR current, the MOF layer seems to facilitate $\mathrm{O}_{2}$ supply, thus making the reagent accessible at the electroactive sites present on the PEI/PEDOT composite. ${ }^{57,66}$ This enhancement becomes even more remarkable if we consider that the MOF is electrochemically inactive, and it could also produce some ionic/electronic transport blocking effects. In this regard, it was recently argued that this modulation effect on the mass and charge transference rates could be the reason for the observed ORR enhancement. ${ }^{77}$ By comparing the integrated charge obtained from the ORR waves for the electrode after each modification step, it is possible to conclude that the enhancement produced by the incorporation of the MOF layer onto the LbL assemblies reaches $55 \%$. Interestingly, this result is the opposite of what was observed when increasing the thickness beyond 4-bilayer film by adding two more bilayers, which yields a $10.6 \%$ decrease. It is relevant to note here that the enhancement obtained upon the deposition of only one layer of MOF yields an increase in $Q_{\mathrm{ORR}}$ which is similar to the one obtained upon the incorporation of $\mathrm{Pt}$ nanoparticles on a conducting polymer film, which has been recently reported by our group. ${ }^{57}$ In this sense, the incorporation of ZIF-8 avoids the use of noble metal catalysts, an expensive and scarce resource, as well as the employment of aggressive and harmful reducing agents which can compromise the polymer structure and performance.

Since it was recently reported that, when employing a $\mathrm{Pt}$ counter electrode (CE) in the electrochemical experiments, $\mathrm{Pt}$ migration from the $\mathrm{CE}$ can occur and the $\mathrm{Pt}$ clusters redeposited onto the working electrode (WE) could result in an artificial higher activity of the evaluated materials, ${ }^{78}$ we also performed the above-mentioned experiments employing a graphite rod as CE. To this end, Au electrodes were modified with a 4-bilayer assembly and the CV curves for the ORR were measured before and after the deposition of a $1 \times$ ZIF-8 layer employing either a $\mathrm{Pt}$ wire or a graphite rod as CE. The measurements are shown in Fig. S3 (ESI $\dagger$ ). It can be observed that the CV curves obtained with graphite CE show very similar ORR current values to those acquired with the Pt CE, and the enhancing effect of the MOF incorporation on the ORR performance is clearly observed in both cases. Therefore, the above possibility can be safely ruled out and we can assign the observed ORR performance improvement solely to the effect of the ZIF-8 MOF layer.

The effect of the amount of MOF deposited on the ORR performance was also explored. Fig. 3D shows the voltammetric response of the $(\mathrm{PEI} / \mathrm{PEDOT})_{4}$ electrodes after " $m$ " successive ZIF-8 deposition cycles ( $m \times$-ZIF-8-modified electrodes). As it can be observed, there is no further enhancement in the cathodic peak of the voltammograms after performing additional growth cycles beyond the first MOF coating. Moreover, the peak current and the charge decrease for $2 \times$ - and $3 \times$-ZIF- 8 films. This effect has been previously observed and it can be ascribed to a less efficient transport of $\mathrm{O}_{2}$ and electrolyte across the MOF coating. ${ }^{57,66}$ ZIF-8 films have been characterized as a 3-D arrange of crystallites exhibiting both hydrophobic intragrain microporosity and intergrain mesoporosity. ${ }^{79}$ The ionic charge transport through ZIF-8 coatings in aqueous environments is purely dominated by solvent-filled intergrain mesoporosity.

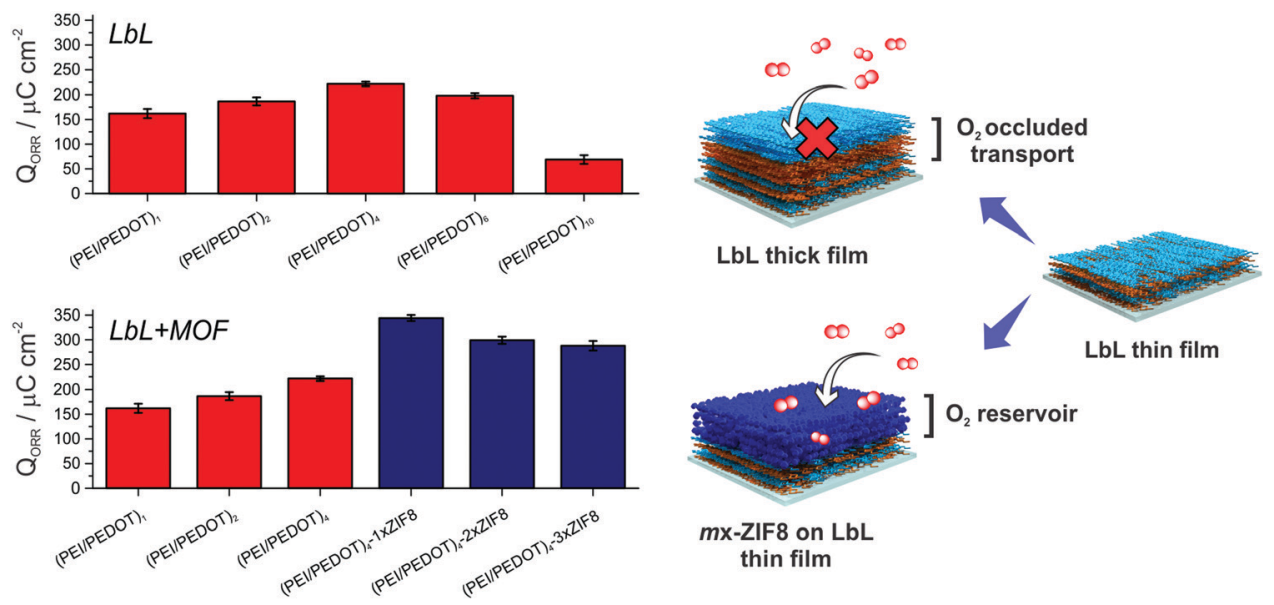

Fig. 4 ORR integrated charge for both strategies $(n=3)$ employed and the schematic representation of the approach. 
As proved using cyclic voltammetry experiments, redox probes are able to diffuse through the solvent-filled pores on the timescale of the electrochemical experiments. ${ }^{80}$ Thus, instead of completely blocking the surface, the MOF coating provides an efficient pathway for molecular oxygen diffusion in the hydrophobic intragrain microporosity; it also allows for the transport of electrolyte ions and solvent in the intergrain mesoporosity. ${ }^{79,80}$ In this regard, the loss of the ORR current when increasing the thickness of the MOF coating can be explained by either a loss in $\mathrm{O}_{2}$ transport efficiency from bulk solution towards the electroactive material through the MOF microporosity, and/or slower transport of ions and solvent in the MOF mesoporosity as the film becomes thicker. Similar results were obtained for increasing potential sweep rates. ${ }^{66}$ Thus, transport limitations in the ORR voltammetric current could appear depending on the experimental conditions; i.e., increasing coating thickness and scan rate.

Furthermore, the enhancement of the ORR current caused by MOF integration was also appreciable in $\mathrm{O}_{2}$-saturated solutions (Fig. 3E). Fig. 4 shows the obtained $Q_{\text {ORR }}$ values for both ORR optimization strategies together with a schematic representation, exhibiting the improvement in the ORR performance of the multilayer electrodes by the developed approach.

\section{Conclusions}

We have presented a rational strategy for the integration of three different building blocks in a functional interfacial nanoarchitecture, improving the properties of the composite film as an electrocatalyst towards ORR. Firstly, multilayer films were produced via the LbL method making use of the electrostatic interactions between the cationic polyelectrolyte PEI and the negatively charged colloidal complex PEDOT:PSS as the counterpart. The growth of the film as the number of bilayers increased was monitored by UV-VIS spectroscopy, showing an exponential growth up to 10 assembled bilayers with an abrupt increase in the amount of material deposited starting from 4 bilayers. While cyclic voltammetry measurements corroborated the electrical connection through the LbL films up to 10 assembled bilayers, the electrochemical performance of the modified electrodes towards the ORR in neutral solutions showed that the integrated charge for the electrocatalytic reaction presents a maximum value for 4 assembled bilayers, and then decreases for 6 and 10 assembled bilayers.

In order to optimize the hybrid electrode material towards ORR, ZIF-8 nanocrystallites were deposited on electrodes previously modified with 4-bilayer LbL assemblies. The micro/mesoporous metal-organic material was found to be chemically and mechanically stable in neutral buffer solutions, and its integration on the electroactive material resulted in an improvement of the ORR performance of the film due to its ability to selectively preconcentrate $\mathrm{O}_{2}$ from solution without occluding the ionic transport. In this way, the ZIF-8 increased the amount of $\mathrm{O}_{2}$ available for the electrochemical reaction that occurs on the PEDOT immersed in the multilayer film, yielding superior ORR performances. It was also found that one deposition cycle of ZIF-8 was sufficient to achieve the maximum increase in the ORR charge, whereas subsequent MOF deposition steps caused a decrease in the electrocatalytic activity of the film.

It is observed that the LbL technique allowed the integration of conducting polymers in the multilayer films in a straightforward way, allowing the thickness and composition of the assemblies to be easily controlled, while preserving electrochemical connectivity throughout the films. In addition to this, the LbL technique allowed the finalization of the assemblies with a layer of PEDOT:PSS, which in turn makes efficient anchoring of ZIF-8 possible due to the affinity towards the exposed sulfonate moieties on the polyanion. Finally, the study illustrates a simple and rapid strategy for the construction and optimization of complex functional interfaces towards ORR in aqueous environments at neutral $\mathrm{pH}$ through the rational incorporation of a conducting polymer in LbL assemblies and the subsequent deposition of a widely used MOF as an in situ porous oxygen reservoir.

\section{Conflicts of interest}

There are no conflicts to declare.

\section{Acknowledgements}

This work was supported by ANPCyT (PICT-2016-1680, PICT2018-0780) and UNLP (PID-X867). GEF acknowledges CONICET for a doctoral fellowship. MR, OA and WAM are CONICET staff members.

\section{References}

1 C. F. Shih, T. Zhang, J. Li and C. Bai, Joule, 2018, 2, 1925-1949.

2 Y. Yang, Y. Yang, Z. Pei, K.-H. Wu, C. Tan, H. Wang, L. Wei, A. Mahmood, C. Yan, J. Dong, S. Zhao and Y. Chen, Matter, 2020, 3, 1442-1476.

3 R. A. Betts, C. D. Jones, J. R. Knight, R. F. Keeling and J. J. Kennedy, Nat. Clim. Change, 2016, 6, 806-810.

4 M. K. Debe, Nature, 2012, 486, 43-51.

5 J. Pan, Y. Y. Xu, H. Yang, Z. Dong, H. Liu and B. Y. Xia, Adv. Sci., 2018, 5, 1700691.

6 A. Kulkarni, S. Siahrostami, A. Patel and J. K. Nørskov, Chem. Rev., 2018, 118, 2302-2312.

7 Z. W. Seh, J. Kibsgaard, C. F. Dickens, I. Chorkendorff, J. K. Nørskov and T. F. Jaramillo, Science, 2017, 355, eaad4998.

8 X. Tian, X. F. Lu, B. Y. Xia and X. W. (David) Lou, Joule, 2020, 4, 45-68.

9 S. Ghosh and R. N. Basu, Nanoscale, 2018, 10, 11241-11280.

10 M. Aono and K. Ariga, Adv. Mater., 2016, 28, 989-992.

11 K. Ariga and Y. Yamauchi, Chem. - Asian J., 2020, 15, 718-728. 
12 K. Ariga and M. Shionoya, Bull. Chem. Soc. Jpn., 2021, 94, 839-859.

13 O. Azzaroni and K. Ariga, Mol. Syst. Des. Eng., 2019, 4, 9-10. 14 J. Luo, R. Liu and X. Liu, J. Mater. Chem. C, 2014, 2, 4818-4827.

15 W. A. Marmisollé and O. Azzaroni, Nanoscale, 2016, 8, 9890-9918.

16 G. E. Fenoy, B. Van der Schueren, J. Scotto, F. Boulmedais, M. R. Ceolín, S. Bégin-Colin, D. Bégin, W. A. Marmisollé and O. Azzaroni, Electrochim. Acta, 2018, 283, 1178-1187.

17 Y. Lvov, K. Ariga, T. Kunitake and I. Ichinose, J. Am. Chem. Soc., 1995, 117, 6117-6123.

18 K. Ariga, J. P. Hill and Q. Ji, Phys. Chem. Chem. Phys., 2007, 9, 2319.

19 S. E. Herrera, M. L. Agazzi, M. L. Cortez, W. A. Marmisollé, C. Bilderling and O. Azzaroni, Macromol. Chem. Phys., 2019, 1900094.

20 A. Vander Straeten, A. Bratek-Skicki, A. M. Jonas, C. A. Fustin and C. C. Dupont-Gillain, ACS Nano, 2018, 12(8), 8372-8381.

21 K. Ariga, Y. Yamauchi, G. Rydzek, Q. Ji, Y. Yonamine, K. C.-W. Wu and J. P. Hill, Chem. Lett., 2014, 43, 36-68.

22 G. E. Fenoy, E. Maza, E. Zelaya, W. A. Marmisollé and O. Azzaroni, Appl. Surf. Sci., 2017, 416, 24-32.

23 G. Rydzek, Q. Ji, M. Li, P. Schaaf, J. P. Hill, F. Boulmedais and K. Ariga, Nano Today, 2015, 10, 138-167.

24 K. R. Knowles, C. C. Hanson, A. L. Fogel, B. Warhol and D. A. Rider, ACS Appl. Mater. Interfaces, 2012, 4, 3575-3583.

25 L. Shao, J.-W. Jeon and J. L. Lutkenhaus, J. Mater. Chem. A, 2014, 2, 14421-14428.

26 T. J. Dawidczyk, M. D. Walton, W. S. Jang and J. C. Grunlan, Langmuir, 2008, 24, 8314-8318.

27 R. M. Iost and F. N. Crespilho, Biosens. Bioelectron., 2012, 31, $1-10$.

28 A. D. Taylor, M. Michel, R. C. Sekol, J. M. Kizuka, N. a. Kotov and L. T. Thompson, Adv. Funct. Mater., 2008, 18, 3003-3009.

29 X. Zhang, X. Zana and Z. Su, J. Mater. Chem., 2011, 21, 17783-17789.

30 D. Banham and S. Ye, ACS Energy Lett., 2017, 2, 629-638.

31 J. G. Ibanez, M. E. Rincón, S. Gutierrez-Granados, M. Chahma, O. A. Jaramillo-Quintero and B. A. FrontanaUribe, Chem. Rev., 2018, 118, 4731-4816.

32 A. Elschner, S. Kirchmeyer, W. Lovenich, U. Merker and K. Reuter, PEDOT: Principles and Applications of an Intrinsically Conductive Polymer, CRC Press, 2010.

33 M. N. Gueye, A. Carella, J. Faure-Vincent, R. Demadrille and J.-P. Simonato, Prog. Mater. Sci., 2020, 108, 100616.

34 S. K. Singh, X. Crispin and I. V. Zozoulenko, J. Phys. Chem. C, 2017, 121, 12270-12277.

35 G. E. Fenoy, C. Bilderling, W. Knoll, O. Azzaroni and W. A. Marmisollé, Adv. Electron. Mater., 2021, 2100059.

36 B. Winther-Jensen, O. Winther-Jensen, M. Forsyth and D. R. MacFarlane, Science, 2008, 321, 671-674.

37 V. Gueskine, A. Singh, M. Vagin, X. Crispin and I. Zozoulenko, J. Phys. Chem. C, 2020, 124, 13263-13272.

38 M. Vagin, V. Gueskine, E. Mitraka, S. Wang, A. Singh, I. Zozoulenko, M. Berggren, S. Fabiano and X. Crispin, Adv. Energy Mater., 2021, 11, 1-8.
39 C. De Saint-Aubin, J. Hemmerlé, F. Boulmedais, M. F. Vallat, M. Nardin and P. Schaaf, Langmuir, 2012, 28, 8681-8691.

40 G. E. Fenoy, O. Azzaroni, W. Knoll and W. A. Marmisollé, Chemosensors, 2021, 9, 212.

41 A. D. Chowdhury, N. Agnihotri, P. Sen and A. De, Electrochim. Acta, 2014, 118, 81-87.

42 G. Gnana kumar, C. Joseph Kirubaharan, D. J. Yoo and A. R. Kim, Int. J. Hydrogen Energy, 2016, 41, 13208-13219.

43 N. B. Muhamad, W. M. Khairul and F. Yusoff, J. Solid State Chem., 2019, 275, 30-37.

44 X. Gao, Int. J. Electrochem. Sci., 2017, 12, 11287-11297.

45 Z. Guo, H. Liu, C. Jiang, Y. Zhu, M. Wan, L. Dai and L. Jiang, Small, 2014, 10, 2087-2095.

46 J. A. Vigil, T. N. Lambert, M. Kelly and R. Aidun, Mater. Chem. Front., 2017, 1, 1668-1675.

47 J. A. Vigil, M. T. Brumbach, J. Duay and T. N. Lambert, RSC Adv., 2018, 8, 24428-24433.

48 S. R. Pering, J. A. Nicholas, B. Bayatsarmadi, D. Evans, M. Fabretto, A. Blencowe, P. J. Murphy and P. Talemi, RSC $A d v .$, 2016, 6, 28809-28814.

49 P. M. Usov, B. Huffman, C. C. Epley, M. C. Kessinger, J. Zhu, W. A. Maza and A. J. Morris, ACS Appl. Mater. Interfaces, 2017, 9, 33539-33543.

50 H. F. Wang, L. Chen, H. Pang, S. Kaskel and Q. Xu, Chem. Soc. Rev., 2020, 49, 1414-1448.

51 Y. Yang, Y. Yang, Y. Liu, S. Zhao and Z. Tang, Small Sci., 2021, 2100015.

52 S. Zhao, C. Tan, C. T. He, P. An, F. Xie, S. Jiang, Y. Zhu, K. H. Wu, B. Zhang, H. Li, J. Zhang, Y. Chen, S. Liu, J. Dong and Z. Tang, Nat. Energy, 2020, 5, 881-890.

53 S. Kuyuldar, D. T. Genna and C. Burda, J. Mater. Chem. A, 2019, 7, 21545-21576.

54 M. Kalaj, K. C. Bentz, S. Ayala, J. M. Palomba, K. S. Barcus, Y. Katayama and S. M. Cohen, Chem. Rev., 2020, 120(16), 8267-8302.

55 X. F. Lu, B. Y. Xia, S. Q. Zang and X. W. Lou, Angew. Chem., Int. Ed., 2020, 59, 4634-4650.

56 J. S. Tuninetti, M. Rafti and O. Azzaroni, RSC Adv., 2015, 5, 73958-73962.

57 G. E. Fenoy, J. Scotto, J. Azcárate, M. Rafti, W. A. Marmisollé and O. Azzaroni, ACS Appl. Energy Mater., 2018, 1, 5428-5436.

58 K. Huang, J. Li, M. Sun, Z. Yang, Y. Fan, X. Du, W. Chen and J. Yang, Appl. Surf. Sci., 2021, 535, 147659.

59 H. Xu, J. Hu, D. Wang, Z. Li, Q. Zhang, Y. Luo, S. Yu and H. Jiang, J. Am. Chem. Soc., 2015, 137, 13440-13443.

60 A. Aijaz, N. Fujiwara and Q. Xu, J. Am. Chem. Soc., 2014, 136, 6790-6793.

61 Q. Liu, Z.-X. Low, L. Li, A. Razmjou, K. Wang, J. Yao and H. Wang, J. Mater. Chem. A, 2013, 1, 11563.

62 Z. Zhang, Y. Chen, X. Xu, J. Zhang, G. Xiang, W. He and X. Wang, Angew. Chem., 2014, 126, 439-443.

63 Z. Li, R. Yu, J. Huang, Y. Shi, D. Zhang, X. Zhong, D. Wang, Y. Wu and Y. Li, Nat. Commun., 2015, 6, 8248.

64 W. T. Koo, S. Qiao, A. F. Ogata, G. Jha, J. S. Jang, V. T. Chen, I. D. Kim and R. M. Penner, ACS Nano, 2017, 11, 9276-9285. 
65 K. S. Park, Z. Ni, A. P. Cote, J. Y. Choi, R. Huang, F. J. UribeRomo, H. K. Chae, M. O'Keeffe and O. M. Yaghi, Proc. Natl. Acad. Sci. U. S. A., 2006, 103, 10186-10191.

66 M. Rafti, W. A. W. A. Marmisollé and O. Azzaroni, Adv. Mater. Interfaces, 2016, 3, 1600047.

67 A. P. Mártire, G. M. Segovia, O. Azzaroni, M. Rafti and W. Marmisollé, Mol. Syst. Des. Eng., 2019, 4, 893-900.

68 Z. Tang, S. T. Donohoe, J. M. Robinson, P. A. Chiarelli and H.-L. Wang, Polymer, 2005, 46, 9043-9052.

69 R. R. Smith, A. P. Smith, J. T. Stricker, B. E. Taylor and M. F. Durstock, Macromolecules, 2006, 39, 6071-6074.

70 D. Wakizaka, T. Fushimi, H. Ohkita and S. Ito, Polymer, 2004, 45, 8561-8565.

71 D. DeLongchamp and P. T. Hammond, Adv. Mater., 2001, 13, 1455-1459.

72 M. Rafti, J. A. Allegretto, G. M. Segovia, J. S. Tuninetti, J. M. Giussi, E. Bindini and O. Azzaroni, Mater. Chem. Front., 2017, 1, 2256-2260.
73 M. Reyes-Reyes, I. Cruz-Cruz and R. López-Sandoval, J. Phys. Chem. C, 2010, 114, 20220-20224.

74 G. M. Segovia, J. S. Tuninetti, O. Azzaroni and M. Rafti, ACS Appl. Nano Mater., 2020, 3, 11266-11273.

75 J. A. Allegretto, A. Iborra, J. M. Giussi, C. von Bilderling, M. Ceolín, S. Moya, O. Azzaroni and M. Rafti, Chem. - Eur. J., 2020, 26, 12388-12396.

76 F. N. Crespilho, F. C. Nart, O. N. Oliveira and C. M. A. Brett, Electrochim. Acta, 2007, 52, 4649-4653.

77 E. C. M. Tse, C. J. Barile, N. A. Kirchschlager, Y. Li, J. P. Gewargis, S. C. Zimmerman, A. Hosseini and A. A. Gewirth, Nat. Mater., 2016, 15, 754-759.

78 R. Chen, C. Yang, W. Cai, H. Y. Wang, J. Miao, L. Zhang, S. Chen and B. Liu, ACS Energy Lett., 2017, 2, 1070-1075.

79 M. I. Velasco, R. H. Acosta, W. A. Marmisollé, O. Azzaroni and M. Rafti, J. Phys. Chem. C, 2019, 123, 21076-21082.

80 J. S. Tuninetti, M. Rafti, A. Andrieu-Brunsen and O. Azzaroni, Microporous Mesoporous Mater., 2016, 220, 253-257. 